\title{
Redefining Cirrhosis - a brief review
}

\author{
Pradhan $\mathrm{SV}^{1}$
}

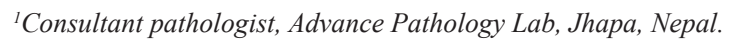

\author{
Keywords: \\ Cirrhosis; \\ Histologic activity index; \\ Ishak; \\ Knodell; \\ Matrix metalloproteinases; \\ Metavir
}

\begin{abstract}
The liver damage is associated with variable amount of fibrosis. The presence of fibrosis with nodule formation is pathognomic of cirrhosis. It is accompanied by vascular remodeling and regeneration with important functional and hemodynamic consequences that include development of portal hypertension and eventually decompensation and death. However fibrosis can regress following successful treatment of the underlying disease. The classification system followed till date does not analyze this aspect. In this brief review the histological features of fibrosis and the newer models for reclassifying cirrhosis is discussed.
\end{abstract}

\section{INTRODUCTION}

"Cirrhosis" derives from the Greek word kíppos, meaning tawny, and it describes the gross finding as tawny nodular and firm. ${ }^{1,2}$ For almost 2 centuries, the emphasis was placed on the irreversible, "end-stage" nature of these livers; etiology was not considered very important because there was no cure, and survival was usually short. In 1977 , an international panel sponsored by the World Health Organization, defined cirrhosis as "a diffuse process characterized by fibrosis and the conversion of normal liver architecture into structurally abnormal nodules."3

The transformation of the normal lobular architecture to the nodule formation in cirrhosis is due to angiogenesis, vascular remodeling, sinusoidal capillarization, perisinusoidal fibrosis, and loss of metabolic zonation. In all these staging systems, cirrhosis constitutes the most advanced stage and often leads to portal hypertension, progressive liver cell failure, decompensation, and death. ${ }^{4,5}$

\footnotetext{
Correspondence:

Dr. S.V . Pradahn, MBBS, MD

Advance Pathology Lab

Birtamode Jhapa

Email: seemavpradhan@gmail.com
}

\section{Fibrosis in chronic liver disease and cirrhosis}

The first histological classification of chronic liver disease was published in 1968 and revised in $1977 .{ }^{6}$ It classified hepatitis into chronic persistent, chronic lobular, and chronic active hepatitis. The classification was only aimed at distinguishing subgroups according to the degree of disease activity and to provide prognostic information and criteria for the use of immunosuppressive therapy. We are now aware of the various etiopathogenesis of liver disease. The natural history, the serological date and recent advances in therapy have been identified. Hence this older classification is not justified. A new categorization of chronic hepatitis is recommended by several authors based upon aetiology, necro-inflammatory activity, and degree of fibrosis.

Assessment of fibrosis can be done best by biopsy of the liver, certain serological parameters, the assessment of hepatic venous pressure gradient (HVPG), "liver stiffness" measurement (based on transient elastography of the liver. ${ }^{7,8}$

The AST/platelet ratio index (APRI) can be calculated from standard blood tests without additional cost. AST is expressed as a function of the upper limit of normal 
(AST/upper normal value for AST $\times 100$ ). As the degree of fibrosis increases, the AST increases with respect to the upper limit of normal and the platelet count declines. As patients develop advanced fibrosis and then cirrhosis, this ratio increases exponentially. Values greater than 1.5 are strongly indicative of advanced fibrosis or cirrhosis. In contrast, the test is unable to differentiate between patients with no or mild fibrosis. However, if the APRI index is increasing consistently over time, it is likely that fibrosis is progressing. The test was developed in patients with chronic $\mathrm{HCV}$ and it remains unclear whether this ratio correlates with fibrosis in other forms of chronic liver disease. ${ }^{9}$

The Fibrotest was developed retrospectively by correlating the liver fibrosis score in patients with chronic HCV to serum biochemical and hematologic markers. Five markers ( $\alpha 2$-macroglobulin, haptoglobin, gamma-glutamyl transpeptidase, total bilirubin, and apolipoprotein $\alpha 1$ ) were selected though multivariate analysis. However all of these biochemical tests are affected by inflammation and that none are precursors of fibrosis. These markers correlate with fibrosis because inflammation increases with the severity of fibrosis in patients with chronic HCV. ${ }^{10}$

The FibroScan is a specialized ultrasound unit that utilizes transient elastography to assess liver fibrosis. Sound waves are sent into the liver and the speed at which these waves return to the transducer is related to the degree of liver stiffness, which increases with increasing fibrosis. The accuracy of FibroScan appears to decline with obesity. ${ }^{10}$

Each of these noninvasive tests of liver fibrosis can accurately differentiate patients with significant fibrosis (more than portal fibrosis) from patients with lesser degrees of fibrosis. However, neither of these tests can accurately differentiate patients with advanced bridging fibrosis and cirrhosis nor differentiate patients with no fibrosis from patients with mild fibrosis. ${ }^{10}$

Liver biopsy is still the best method for the assessment of fibrosis in liver. The adequacy of the sample is important to assess the biopsy. There are various definitions of adequacy in liver biopsies. The recommended size is $20 \mathrm{~mm} \times 1 \mathrm{~mm}$ with 11 portal tracts is enough to grade chronic activity in liver.

The assessment of liver biopsy is assisted by various scoring system. The history of these scoring systems dates back to 1981 when the histological features of chronic hepatitis were evaluated for determining the prognosis according to the pathophysiology of chronic hepatitis B virus (HBV) infection, and organised into a scoring system by Knodell and colleagues. ${ }^{11}$

\section{Metavir scoring system ${ }^{12}$}

The Metavir scoring system was specially designed for patients with hepatitis $\mathrm{C}$. The scoring consists of using a grading and a staging system. The grade gives an indication of the activity or amount of inflammation and the stage represents the amount of fibrosis or scarring.

The grade is assigned a number based on the degree of inflammation, which is usually scored from $0-4$ with 0 being no activity and 3 or 4 considered severe activity. The amount of inflammation is important because it is considered a precursor to fibrosis.

The fibrosis score is also assigned a number from 0-4:

- $0=$ no scarring

$\cdot 1=$ minimal scarring

- 2 = scarring has occurred and extends outside the areas in the liver that contains blood vessels

$\cdot 3$ = bridging fibrosis is spreading and connecting to other areas that contain fibrosis

- $4=$ cirrhosis or advanced scarring of the liver

\section{Knodell Scoring system ${ }^{11}$}

The Knodell score or histologic activity index (HAI) is also commonly used to stage liver disease. It a somewhat more complex process, but some experts believe that it is a better tool for defining the extent of liver inflammation and damage. It is composed of four individually assigned numbers that make up a single score.

The first component (perioportal and/or bridging necrosis) is scored $0-10$.

The next two components (intralobular degeneration and portal inflammation) are scored 0-4.

The combination of these three markers indicates the amount of inflammation in the liver:

- 0 = no inflammation

-1-4 = minimal inflammation

- 5-8 = mild inflammation

- 9-12 = moderate inflammation

-13-18 = marked inflammation

The fourth component indicates the amount of scarring in the liver and is scored from 0 (no scarring) to 4 (extensive scarring or cirrhosis).

The currently most widely used system is the Ishak, or "revised Knodell", system which attempts to correct the criticism of numerical discontinuity by reintroducing the number $2 .{ }^{13}$

Information about the grade and stage of liver disease is helpful for the health care provider and the patient in guiding medical management. Various histological score are compared in the following table no. 1. 
Table 1: Liver Histology scoring system

\begin{tabular}{|c|c|c|c|c|}
\hline Description & Knodel & Metavir ${ }^{12}$ & Ishak $^{14}$ & Scheuer ${ }^{15}$ \\
\hline No fibrosis & 0 & 0 & 0 & 0 \\
\hline Portal fibrosis & 1 & 1 & 1 and 2 & 1 \\
\hline Portal fibrosis with septae extending outside portal area & & 2 & 2 and 3 & 2 \\
\hline Bridging fibrosis & 3 & 3 & 3 and 4 & 3 \\
\hline Cirrhosis & 4 & 4 & 5 and 6 & 4 \\
\hline
\end{tabular}

The uses of these scoring systems increase the homogenous interpretation of liver biopsy by increasing reproducibility. However the assessment of fibrosis can be subjective and vary according to the observer. This can be improvised by use of histochemical stains. The commonly used stains include elastic trichome stain and reticulin stains. They help in identifying the fibrosis but collagen deposition is best appreciated and is reproducible by Sirius stain.

Assessment of fibrosis is better appreciated and reproducible in Sirius red stain. Ishak score ${ }^{6,14}$ has been illustrated in the following table 2 .

In recent years, quantitative evaluations through image analysis have been utilized to assess fibrosis in liver. Digital image analysis rapidly provides objective quantitative results similar to but more precise than those determined by semi-quantitative scoring methods.

There are various methods for digital analysis. The one that has more sensitivity is the fibroquant method. ${ }^{16}$ In this method the area of fibrosis are measured dividing the different areas of fibrosis. The perisinusoidal fibrosis areas (SF), portal-periportal and septal fibrosis areas (PF), portalperiportal and septal area (PA), and portal vessel and biliary duct lumen areas (LA) are automatically quantified in, mn' and shown superimposed on the initial preprocessed image. Percentage area values are also calculated, as follows: ${ }^{16}$

- Percentage area of perisinusoidal fibrosis (PSF):

$\mathrm{PSF}=1 \mathrm{OO} * \mathrm{SF} /$ (IA-PA), where IA is the area of the whole image;

- Percentage area of portal-periportal and septal fibrosis (PPF): $\mathrm{PPF}=1 \mathrm{OO} * \mathrm{PF} /(\mathrm{PA}-\mathrm{LA})$;

- Percentage area of portal vessel and biliary duct lumina (PLA): PLA=1OO+LA/PA.

FibroQuant image processing is a very sensitive and reproducible method of fibrosis quantiflcation. The digita image analysis complements semiquantitative histologic evaluation systems and matches with level of HPVG and liver stiffness measurement. ${ }^{16}$

\section{Evolution of cirrhosis - regression of Fibrosis}

Cirrhosis was considered irreversible and was often referred

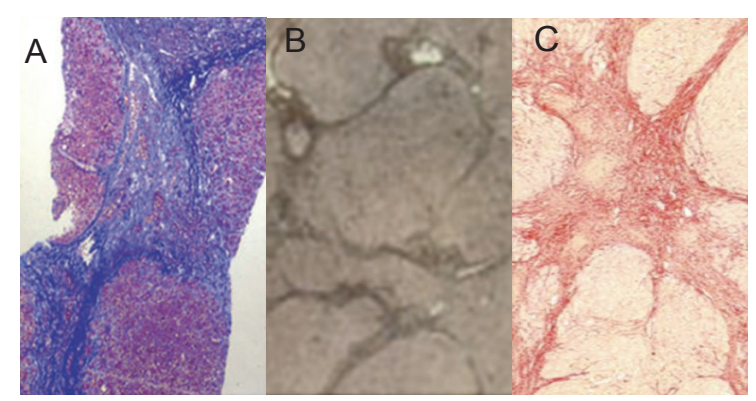

Figure 1: Fibrosis Demonstated by various histochemical stains (A. Masson Trichrome, B. Reticulin stain, C. Sirius red $(X 100)$.

Table 2: Ishak score ${ }^{6,14}$ with illustrated diagram.

\begin{tabular}{|c|c|c|c|}
\hline Appearance & $\begin{array}{l}\text { Ishak stage: } \\
\text { Categorical description }\end{array}$ & $\begin{array}{l}\text { Ishak stage: } \\
\text { Categorical } \\
\text { assignment }\end{array}$ & $\begin{array}{l}\text { Fibrosis } \\
\text { measurement }\end{array}$ \\
\hline & No fibrosis (normal) & 0 & $1.9 \%$ \\
\hline * & $\begin{array}{l}\text { Fibrous expansion of some portal } \\
\text { area }+/ \text { - short fibrous septa }\end{array}$ & 1 & $3.0 \%$ \\
\hline & $\begin{array}{l}\text { Fibrous expansion of most portal areas } \\
+/ \text { - short fibrous speta }\end{array}$ & 2 & $3.6 \%$ \\
\hline & $\begin{array}{l}\text { Fibrous expansion of most portal areas } \\
\text { with occasional } P \text {-P bridging }\end{array}$ & 3 & $6.5 \%$ \\
\hline & $\begin{array}{l}\text { Fibrous expansion of portal areas with } \\
\text { marked bridging ( } P \text { - } P \text { as well as } P-C)\end{array}$ & 4 & $13.7 \%$ \\
\hline & $\begin{array}{l}\text { Marked bridging with occasional } \\
\text { nodules (incomplete cirrhosis) }\end{array}$ & 5 & $24.3 \%$ \\
\hline & Cirrhosis, probable or definite & 6 & $27.8 \%$ \\
\hline
\end{tabular}

*Proportion (\%) of area of illustrated section showing Sirius red staining for collagen (collagen proportionate area)

to as end-stage liver disease. Recent studies and researches however showed that liver fibrosis and remodeling is a dynamic process and even cirrhosis can regress. The decrease in the fibrosis is mainly caused by ECM enzymatic digestion. The matrix metalloproteinases (MMPs) efficiently cleave collagens and other components of the extracellular matrix. This enzyme family is composed of different calcium-dependent and zinc dependent enzymes, each being specific for a group of ECM components, interstitial collagenases (MMP-1, MMP-8), gelatinases (MMP-2, MMP-9), stromelysins (MMP-3, MMP-7, MMP10, MMP-11), membrane type (MMP-14, MMP-15, MMP16, MMP-17, MMP-24, MMP-25), and metalloelastases 


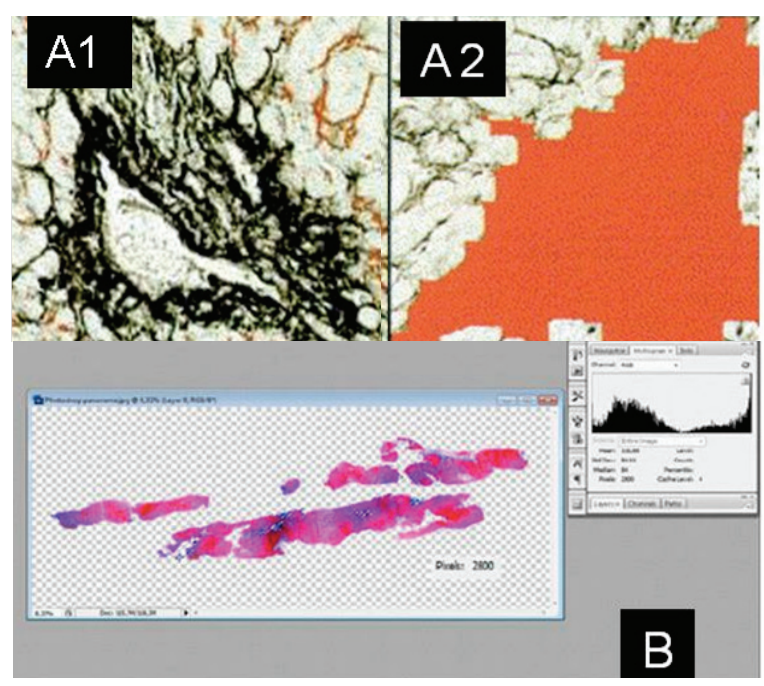

Figure 3: Digital image analysis of Fibrosis. Analysis A. Areas quantified by fibroquant A1- Image of liver tissue with Sirius red, A2-portal periportal area. B. Analysis of area by photoshop.

(MMP-12). Increased collagenase activity is a primary pathway of fibrosis resolution. ${ }^{17,18}$

Withdrawal of the causal source of the chronic injury (eg, HBV, HCV) results in decreased ECM production and increased collagenase activity responsible for matrix degradation. The cellular origin of MMP production during liver fibrosis has been assigned mainly to hepatic stellate cells and activated macrophages/ Kupffer cells. MMP activation is balanced by an inactivation mechanism, namely the binding, in a specific ratio, to a group of inhibitors known as tissue inhibitors of metalloproteinases (TIMPs). ${ }^{17,18}$

During fibrosis regression, increased activity of collagendegrading enzymes correlates with decreased TIMPs. ${ }^{18}$

Once ECM has been degraded, activated hepatic stellate cells (HSCs) progressively disappear. HSCs are considered to play a major role in the development of liver fibrosis. HSCs are perisinusoidal cells that normally reside in the space of Disse and contain numerous retinoid and lipid droplets. In response to injury, quiescent HSCs down regulate retinoid storage, acquire contractility, and get transformed into collagen type I1 a-SMA1 (Smooth muscle action) myofibroblasts. ${ }^{3,8}$ Resolution of stellate cell activation represents an essential step toward reversibility of fibrosis. There is strong evidence that liver cell regeneration is also a crucial condition needed for cirrhosis to regress. Morphologic studies suggest that the succession from micronodular to macronodular cirrhosis represents the early anatomic step in cirrhosis regression in humans. ${ }^{8,9}$ In this scheme, it is postulated that regeneration of liver cells acts through distension and rupture of fibrous septa leading to a partial recovery of the lobular architecture. The functional demonstration of liver cell regeneration as a driver of cirrhosis regression has been shown in an animal model of liver cirrhosis in which hepatocyte regeneration has been forced through telomerase gene delivery in liver cells. An efficient treatment of disorders such as antiviral drug therapy for viral hepatitis, allows the arrest of necroinflammation and liver regeneration to occur, mechanism that may favor cirrhosis regression. Reversibility of fibrosis depends on the age of the accumulated fibrillar ECM. ${ }^{19}$

\section{Shall we redefine cirrhosis??}

The old classification includes the Ishak, Batts and Ludwig, or METAVIR staging chronic liver disease. ${ }^{6,12,20}$ The fibrosis in cirrhosis was graded accordingly. However the concept is evolving now with new theories of regression of fibrosis being noted. It is necessary to stage the degree of fibrosis within a cirrhotic liver.

\section{HISTOPATHOLOGIC PATTERN OF REGRESSING CIRRHOSIS}

Cirrhosis regression is associated with a net decrease in the amount of fibrous tissue that can easily be shown by morphometric measurement of fibrosis area. The detection of histologic features suggestive of regression may represent useful information; with therapeutic and prognostic implications, provided a detailed account of such regressive changes, which they termed "hepatic repair complex." ${ }^{21}$

\section{Histologic features of regressed cirrhosis: ${ }^{21}$}

- No shunting vessels expanding from portal tract

- Partial or full restoration of lobular architecture

- Some of the portal tract or central vein visible

- Lack of ductular proliferation

- Restoration of enzymatic lobular zonation

There is decrease in fibrosis with residual few collagen bundles only. Due to reduction in angiogenesis and fibrogenesis, there is disappearance of shunting neovessels that develop as a result of angiogenesis associated with fibrogenesis. Thin septa of regressing cirrhosis usually lack vessels or contain only a few residual capillaries that may not function as shunting vessels. Thereafter, septa become incomplete (incomplete septal cirrhosis) or may disappear (fig.4). The rounded septal contours and aligned ends of septa suggest that complete cirrhosis may have been present in the past, although this cannot be known with certainty.

Regression of cirrhosis leads to partial or full restoration of the lobular organization. The portal tracts and central veins are present, some of them containing an incomplete portal triad. Portal vein thrombosis may halt this reshaping of lobular organization.

Nodular organization may persist despite the disappearance of fibrous septa leading to a pattern that closely resembles nodular regenerative hyperplasia ${ }^{15}$ 

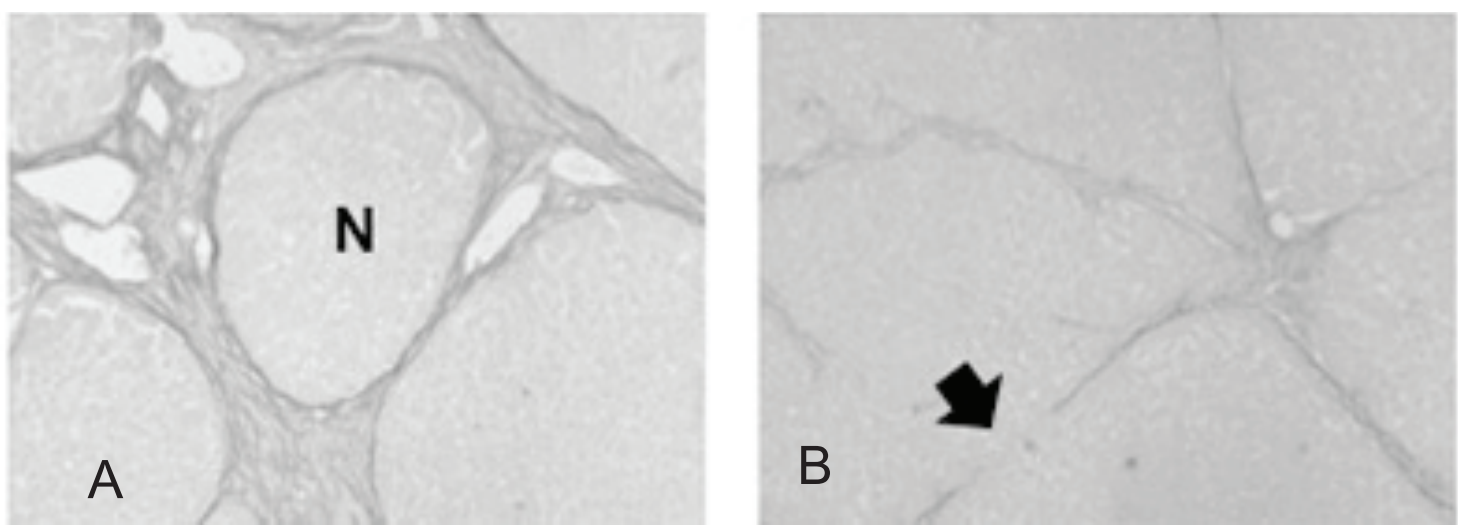

Figure 4: A. Cirrhosis with nodules and thick fibrous septa. B. Perforated (incomplete) septa after regression of fibrosis (arrow).

Table 3: Laennec system ${ }^{7}$

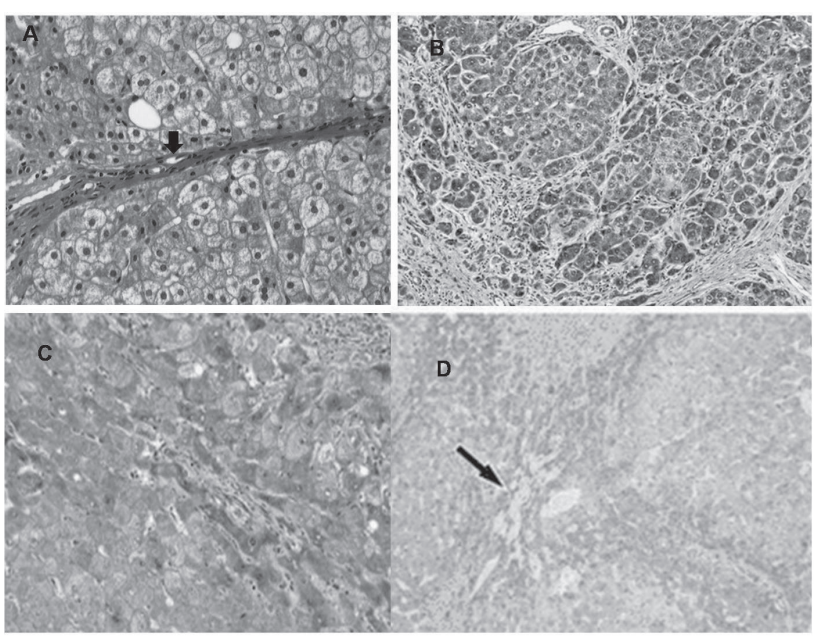

Figure 5: Features of regression of fibrosis. A. Thinning of fibrous septa (H\&E). B. Sinusoidal fibrosis is displaced by expanding clusters of hepatocytes. (elastic trichrome, X140). C. Incomplete cirrhosis. Remnant of a portal tract with arteries and duct, but no portal vein. (Elastic trichrome,X150). D. A region of dark hepatocytes containing delicate remains of septa along with telangiectic sinusoids (arrow) (Elastic trichrome, X65).

\begin{tabular}{llll}
\hline Mild cirrhosis & Moderate cirrhosis & Severe cirrhosis \\
\hline $\begin{array}{l}\text { Definite or probable } \\
\text { cirrhosis, } 1 \text { broad }\end{array}$ & $\begin{array}{l}\text { but not very wide } 2 \text { broad septa } \\
\text { beptum allowed. }\end{array}$ & $\begin{array}{l}\text { Very wide septa } \\
\text { and less then half of one) or }>1 / 2 \\
\text { the biopsy with small } \\
\text { nodule. }\end{array}$ & $\begin{array}{l}\text { minute nodules. } \\
\text { mith }\end{array}$ \\
&
\end{tabular}
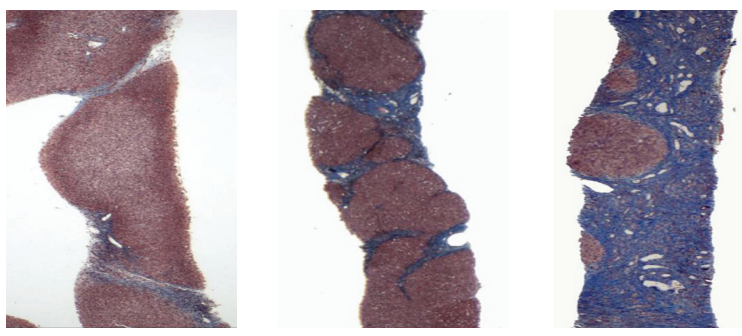

There are isolated, thick collagen fibers; delicate periportal fibrous spikes; portal tract remnants; hepatic vein remnants with prolapsed hepatocytes; hepatocytes within portal tracts or splitting septa (fig.5) minute regenerative nodules; and aberrant parenchymal veins. Ductular proliferation, a feature associated with fibrosis progression, quickly disappears in regressing cirrhosis. In addition, although metabolic zonation is generally lost in cirrhosis, reversion is associated with restitution of normal lobular enzymatic

Table 4: Proposed classification of cirrhosis

\begin{tabular}{|c|c|c|c|}
\hline $\mathbf{S} / \mathbf{N}$ & $4 a$ & $4 b$ & $4 c$ \\
\hline \multirow[t]{2}{*}{ Clinical } & Compensated cirrhosis & & Decompensated cirrhosis \\
\hline & No varices & varices & $\begin{array}{c}\text { Variceal haemorrhage, encephalopathy, } \\
\text { ascitis }\end{array}$ \\
\hline HPVG (mmHg) & $6-10$ & $10-12$ & $>12$ \\
\hline Histological score & $<4$ & 4 & $>4$ \\
\hline Fibrosis (\%) & $<10$ & $10-30$ & $>30$ \\
\hline \multicolumn{2}{|r|}{ Score : septae } & \multicolumn{2}{|c|}{ Score - Nodules } \\
\hline \multicolumn{2}{|c|}{$\begin{array}{l}\text { Thin septae }(<0.1 \mathrm{~mm})=1 \\
\text { Intermedait septae }(0.1-0.2 \mathrm{~mm})=2 \\
\text { Thick septae }(>0.2 \mathrm{~mm})=4\end{array}$} & \multicolumn{2}{|c|}{$\begin{array}{l}\text { Large nodules }(>2 \mathrm{~mm})=1 \\
\text { Mixed nodule }=2 \\
\text { Small nodule }(, 1 \mathrm{~mm}) \mathrm{v}=3\end{array}$} \\
\hline
\end{tabular}




\section{zonation. $^{21}$}

\section{Regression of fibrosis and scoring system}

In view of the regression of fibrosis the Laennecs classification is best adopted as it analyses the thickness of the septa and small nodules. This classification has been called the Laennec system ${ }^{7}$ (Table 3) for the staging of cirrhosis into 3 subcategories of the METAVIR cirrhotic stage (stage 4), as follows: a) Mild cirrhosis b) Moderate cirrhosis c) Severe cirrhosis. (Table 3)

Septa were defined as broad when the thickness was equivalent to the size of the nodules and as very broad when the thickness was greater than the size of the nodules. Later Leanace system was reclassified with addition of histological features. The proposed and revised classification includes all the factors including fibrosis, HPVG, features of compensation (Table 4). ${ }^{22,23}$

Keeping in view the clinical spectrum, the natural course, identification of features of fibrosis and its regression all liver biopsy of chronic liver disease must be assessed including the etiology, the stage, activity of disease process and risk for malignancy.

Additional studies may further clarify the histologic features that are predictive of progression or regression of each chronic liver disease, thus aiding clinicians in patient management.

\section{REFERENCES}

1. Cotran RS, Kumar V, Robbins SL: Robbins Pathologic Basis of Disease. 5th ed. W.B. Saunders, Philadelphia. 1994,834pp.

2. Rosai Juan. Rosai and Ackerman's Surgical Pathology. Vol 1, 9th ed; Elsevier Inc, 2004. 934pp.

3. Kasper DL, Braunwald E, Fauci AS, Hauser SL, Longo DL, Jameson JL. Harrison's Principles of Internal Medicine, 16th Ed: McGraw-Hill, New York: 1994.1844pp.

4. Okudaira M, Atari E Oubu M. Liver cirrhosis, its definition and classification--from a morbid anatomical point of view. Nihon Rinsho 1994;52:5-10.

5. Kamegaya K. Definition and classification of liver cirrhosis. Nihon Rinsho1994;52:11-8.

6. RAnthony PP, Ishak KG, Nayak NC et al. The morphology of cirrhosis. Recommendations on definition, nomenclature, and classification by a working group sponsored by the World Health Organization. J Clin Pathol 1978;31:395-414.
7. Rastogi A, Maiwall R, Bihari $\mathrm{C}$ et al.Cirrhosis histology and Laennec staging system correlate with high portal pressure; Histopathology 2013;62:731-4.

8. Castera L, Vergniol J, Foucher J et al.Prospective comparison of transient elastography, Fibrotest, APRI, and liver biopsy for the assessment of fibrosis in chronic hepatitis C. Gastroenterology 2005; 128:343-50.

9. 9 Wai CT, Greenson JK, Fontana RJ et al. A simple noninvasive index can predict both significant fibrosis and cirrhosis in patients with chronic hepatitis C. Hepatology 2003;38:518-26.

10. Castera L, Vergniol J, Foucher J et al. Prospective comparison of transient elastography, Fibrotest, APRI, and liver biopsy for the assessment of fibrosis in chronic hepatitis C. Gastroenterology. 2005;128:343-50.

11. Knodell RG, Ishak KG, Black WC et al. Formulation and application of a numerical scoring system for assessing histological activity in asymptomatic chronic active hepatitis. Hepatology 1981;1:431-5.

12. Poynard T, Bedossa P, Opolon P. Natural history of liver fibrosis progression in patients with chronic hepatitis $\mathrm{C}$. The OBSVIRC, METAVIR, CLINIVIR, and DOSVIRC groups. Lancet 1997;349:825-32.

13. Standish RA, Cholongitas E, Dhillon A et al. An appraisal of the histopathological assessment of Liver fibrosis. Gut 2006;55;569-78.

14. Ishak K, Baptista A, Bianchi L et al. Histopathological grading and staging of chronic hepatitis. J Hepatol 1995;22:696-9

15. Scheuer PJ. Classification of chronic viral hepatitis: a need for reassessment. J Hepatol 1991;13:382-4

16. Masseroli M, Caballero T, O'Valle F, Del Moral RM, Pérez-Milena A, Del Moral RG. Automatic quantification of liver fibrosis design and validation of a new image analysis method: comparison with semiquantitative indexes of Fibrosis; J Hepatol 2000;32:453-64.

17. Arthur MJ. Collagenases and liver fibrosis; Hepatol 1995;22:43-8.

18. Iredale JP. Hepatic stellate cell behavior during resolution of liver injury. Semin Liver Dis 2001;21:427-36.

19. Garcia-Tsao G, Friedman S, Iredale $J$ et al. Now There Are Many (Stages) Where Before There Was One: In Search of a Pathophysiological Classification of Cirrhosis. Hepatology 2010;51:1445-9.

20. Batts KP, Ludwig J. Chronic hepatitis an update on terminology and reporting. Am J Surg Pathol 1995;19:1409-1417.

21. Hytiroglou P, Snover DC, Alves V et al. Beyond "cirrhosis": a proposal from the International Liver Pathology Study Group. Am J Clin Pathol 2012;137:5-9.

22. Bedossa P, Garcia-Tsao G, Jain D. Cirrhosis Regression and Subclassification; Hepatology 2007;45:886-94.

23. Nagula S, Grosszmann RJ. histological-haemodynamic correlation in cirrhosis- a histological classification of severity of cirrhosis. J.Hepatol 2006;44;111-7. 